\title{
Solvent-free one-pot efficient and highly regioselective access to functionalized thiazolopyridones from $\alpha$-enolic dithioesters
}

\author{
Anugula Nagaraju, B. Janaki Ramulu, Gaurav Shukla, Abhijeet Srivastava, \\ Girijesh Kumar Verma, and Maya Shankar Singh* \\ Department of Chemistry, Faculty of Science, Banaras Hindu University, Varanasi-221005, \\ India \\ E-mail:mayashankarbhu@gmail.com
}

\section{Dedicated to Professor (Dr.) J. S. Yadav on the occasion of his $65^{\text {th }}$ birthday}

\section{DOI: http://dx.doi.org/10.3998/ark.5550190.p009.179}

\begin{abstract}
An operationally simple, highly convergent and straightforward synthesis of diverse thiazolopyridones has been achieved via three-component domino coupling (3CDC) of $\alpha$-enolic dithioesters, cysteamine and dialkyl acetylenedicarboxylates under solvent-free conditions. The approach is carbon-economic and relies on sequential cyclic $N, S$-acetal formation/Michael addition/N-cyclization cascade forming consecutive C-C, C-N and C-S bonds in one-pot.
\end{abstract}

Keywords: Thiazolopyridones, $\alpha$-enolic dithioesters, domino coupling, cascade reactions, solvent-free conditions

\section{Introduction}

The key goal in modern organic synthesis is to design and develop new synthetic strategies for the rapid generation of function-oriented molecules by improving resource efficiency that provides maximum structural diversity and complexity with step, atom, and cost economy. Cascade processes that incorporate multiple bond-forming events in one-pot have come into play, and are of paramount interest in organic synthesis. ${ }^{1-4}$ The synthesis of heterocycles has always been a key aspect, and increasingly attracted the synthetic pursuit of chemists. They are vital part of new drug discovery and indispensable materials in the implementation of any industrial evolution. ${ }^{5-7}$

Among the fused $N, S$-heterocyclic frameworks, thiazolopyridones are versatile privileged scaffolds present in many biologically important products and pharmaceuticals. They exhibit activities like DNA gyrase $\mathrm{B}$ inhibitors ${ }^{8,9}$ and antibacterial agents. ${ }^{10,11}$ Furthermore, 
thiazolopyridones have also been utilized in the synthesis of natural-product-like heterocycles. ${ }^{12}$ Thiazolo ring-fused 2-pyridones have proven to be highly interesting scaffolds for the development of biologically active compounds particularly introducing a variety of substituents in the 2-pyridone part of the heterocycles. ${ }^{13}$ Further, thiazolofused 2-pyridones have been halogenated with complete regioselectivity to synthesize 6-bromo-8-iodo-substituted bicyclic 2pyridones in gram scale. ${ }^{14}$ Recently, a selective intramolecular 5-exo-dig or 6-endo-dig cyclization enroute to 2-furanone or 2-pyrone containing tricyclic scaffolds have been developed. ${ }^{15}$ An efficient method to synthesize a novel rigid tricyclic peptidomimetic scaffold through ring-closure of amino-functionalized bicyclic 2-pyridones has been discovered. ${ }^{16}$

Among available reports for the synthesis of benzothiazolopyridones, ${ }^{17-20}$ most of them suffer from significant limitations such as harsh reaction conditions, expensive catalysts / reagents, prolonged reaction times, multistep syntheses and poor availability of starting materials. In combination, the above facts prompted us to develop a new straightforward and widely applicable approach for the synthesis of thiazolopyridones. The carbon-sulfur and carbonnitrogen bond formations constitute a very important class of reactions in biological processes. In view of the spectacular role of domino reaction in chemical transformations, we are interested in developing metal-free and solvent-free multicomponent reactions to construct complex molecules from readily available simple precursors in a single operation. $\alpha$-Enolic dithioesters are valuable synthetic targets due to their versatile reactivity as powerful intermediates toward the synthesis of various heterocycles. ${ }^{21-25}$ Solvent-free MCRs of $\alpha$-enolic dithioesters are particularly intriguing and have attracted remarkable interest. In continuation of our research interests regarding the synthetic utility of $\alpha$-enolic dithioesters, ${ }^{26-31}$ particularly aimed at exploring one-pot solvent-free synthetic protocols, ${ }^{32-38}$ we report herein an operationally simple and expedient one-pot synthesis of thiazolopyridones under metal-free and solvent-free conditions via one-pot multicomponent domino reaction (MDR) involving a sequence of cyclic $\mathrm{N}, \mathrm{S}$-acetal formation/Michael addition/N-cyclization cascade in good to excellent yield (Scheme $1)$.

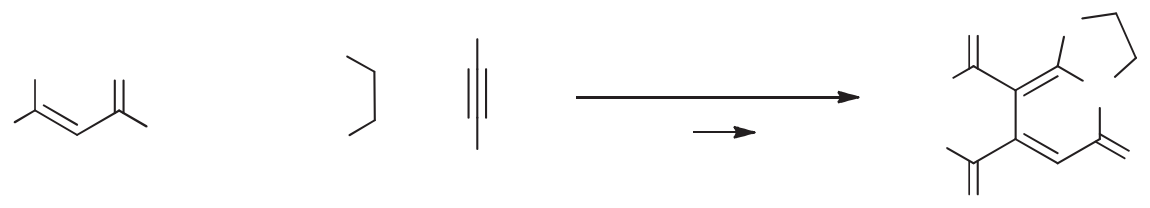

Scheme 1. Synthesis of thiazolopyridones 4 .

\section{Results and Discussion}

To the best of our knowledge, no report on the synthesis of thiazolopyridones utilizing $\alpha$-enolic dithioesters is known to date. To optimize the reaction condition for the synthesis of thiazolopyridones, methyl 3-hydroxy-3-phenyl-prop-2-enedithioate (1a), cysteamine (2) and diethyl acetylenedicarboxylate (3a) were taken as model substrates. We performed the model 
reaction under varying conditions, and the results are listed in Table 1. In an initial study, 1a upon treatment with $\mathbf{2}$ gave cyclic $N, S$-acetal, which has been isolated and fully characterized. ${ }^{39}$ Subsequent treatment of $N, S$-acetal $\mathbf{A}$ with 3a in refluxing methanol afforded the desired thiazolopyridone $\mathbf{4 a}$ in $85 \%$ yield within $4 \mathrm{~h}$. Next to check the influence of solvents, we screened various solvents such as polar protic, low boiling polar aprotic and high boiling polar aprotic solvents (Table 1, entries 2-8). The high boiling polar aprotic solvents displayed better results (Table 1, entries 6 and 7). In one attempt, we tried the reaction under sonication at $40{ }^{\circ} \mathrm{C}$ by adding 2 drops of ethanol for homogenization of reaction mixture. Work up of the reaction afforded the desired product in $85 \%$ yield within $3 \mathrm{~h}$. Finally, we performed the model reaction under solvent-free conditions at $110{ }^{\circ} \mathrm{C}$. Satisfyingly, the reaction completed within $10 \mathrm{~min}$ consuming the reactants completely and furnished the desired product in $63 \%$ yield (Table 1 , entry 10). In order to make the reaction milder, we reduced the temperature after formation of cyclic $N, S$-acetal to $90{ }^{\circ} \mathrm{C}$, when the desired product was obtained in $87 \%$ yield within 30 min (Table 1, entry 11). Further lowering in temperature could not improve the result (Table 1, entry 12). Thus, the best reaction conditions for the formation of $4 \mathbf{a}$ was found to be $\mathbf{1 a}(1 \mathrm{mmol}), \mathbf{2}(1$ mmol), 3a (1 mmol), at $90{ }^{\circ} \mathrm{C}$ under solvent-free condition (Table 1, entry 11).

Table 1. Optimization studies for the synthesis of thiazolopyridone ${ }^{a} \mathbf{4 a}$

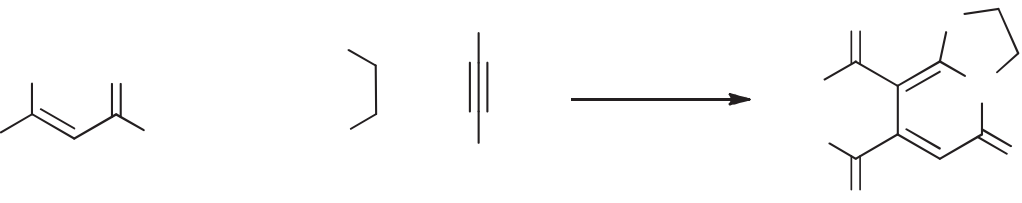

\begin{tabular}{|c|c|c|c|c|}
\hline Entry & Solvent & Temp $\left({ }^{\circ} \mathrm{C}\right)$ & Time (h) & Yield $^{b}(\%)$ \\
\hline 1 & Methanol & Reflux & 4.0 & 85 \\
\hline 2 & Ethanol & Reflux & 3.5 & 80 \\
\hline 3 & $\mathrm{DCM}$ & Reflux & 4.5 & 70 \\
\hline 4 & Chloroform & Reflux & 4.0 & 72 \\
\hline 5 & Acetonitrile & Reflux & 5.0 & 75 \\
\hline 6 & DMF & 110 & 1.5 & 80 \\
\hline 7 & DMSO & 110 & 1.0 & 80 \\
\hline 8 & Water & 100 & 24 & trace \\
\hline 9 & $\begin{array}{c}\text { Ethanol, } 2 \\
\text { drops }\end{array}$ & 40 (sonication) & 3.0 & 85 \\
\hline 10 & none & 110 & $10 \mathrm{~min}$ & 63 \\
\hline 11 & none & 90 & $30 \mathrm{~min}$ & 87 \\
\hline 12 & none & 80 & 1.0 & 70 \\
\hline
\end{tabular}

${ }^{a}$ All reactions were performed with $\mathbf{1 a}(1 \mathrm{mmol}), 2(1 \mathrm{mmol})$, and $\mathbf{3 a}(1 \mathrm{mmol}) ;{ }^{\mathrm{b}}$ Isolated pure yields. 
Experiments probing the scope and generality of this protocol under optimized conditions are summarized in Table 2. A broad range of $\alpha$-enolic dithioesters $\mathbf{1}$, bearing $\mathrm{R}^{1}$ as aryl, hetaryl, and extended aromatic groups, were tolerated well. Dialkyl acetylenedicarboxylates $\mathbf{3}$ bearing both methyl and ethyl as $\mathrm{R}^{2}$ could be employed to afford thiazolopyridones $\mathbf{4}$ in good to excellent yields. All reactions proceeded smoothly and afforded the corresponding product 4 in high yield. A range of $\alpha$-enolic dithioesters bearing $\mathrm{R}^{1}$ as aryl groups with electron-donating substituents were well tolerated, and gave considerably higher yields than those with electron-withdrawing group (4c-f vs. 4g). Moreover, halogen substitution on the $\mathrm{R}^{1}$ of dithioester did not disturb the reactivity, and the corresponding products were formed in high yields $(\mathbf{4 h}, \mathbf{i})$. Importantly, dithioester 1 bearing a heteroaromatic moiety at $\mathrm{R}^{1}$ was also compatibly providing high yield of the product $(\mathbf{4 j} \mathbf{j} \mathbf{l})$. After successful utilization of aromatic dithioesters, we next extended our study to various extended aromatics such as 1-naphthyl $\mathrm{R}^{1}$ substituent, which was also tolerated well and furnished the product $(\mathbf{4 m})$ in good yield.

Table 2. Scope of substrate for the synthesis of thiazolopyridones ${ }^{a} 4$

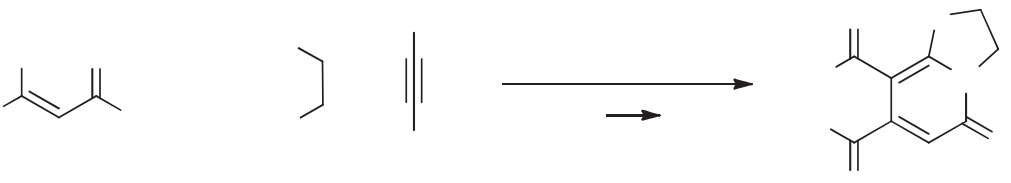

\begin{tabular}{ccccc}
\hline Product & $\mathrm{R}^{1}$ & $\mathrm{R}^{2}$ & $\begin{array}{c}\text { Time } \\
(\mathrm{min})\end{array}$ & $\begin{array}{c}\text { Yield }^{b} \\
(\%)\end{array}$ \\
\hline $\mathbf{4 a}$ & $\mathrm{Ph}$ & $\mathrm{Et}$ & 30 & 87 \\
$\mathbf{4 b}$ & $\mathrm{Ph}$ & $\mathrm{Me}$ & 40 & 80 \\
$\mathbf{4 c}$ & $4-\mathrm{MeC}_{6} \mathrm{H}_{4}$ & $\mathrm{Et}$ & 30 & 92 \\
$\mathbf{4 d}$ & $4-\mathrm{MeOC}_{6} \mathrm{H}_{4}$ & $\mathrm{Et}$ & 45 & 82 \\
$\mathbf{4 e}$ & 3- $^{-\mathrm{MeOC}_{6} \mathrm{H}_{4}}$ & $\mathrm{Me}$ & 45 & 85 \\
$\mathbf{4 f}$ & 1,3-benzodioxol-5-yl & $\mathrm{Me}$ & 35 & 90 \\
$\mathbf{4 g}$ & 4- $\mathrm{F}_{3} \mathrm{CC}_{6} \mathrm{H}_{4}$ & $\mathrm{Me}$ & 55 & 75 \\
$\mathbf{4 h}$ & $2-\mathrm{ClC}_{6} \mathrm{H}_{4}$ & $\mathrm{Et}$ & 50 & 75 \\
$\mathbf{4 i}$ & $2-\mathrm{ClC}_{6} \mathrm{H}_{4}$ & $\mathrm{Me}$ & 45 & 70 \\
$\mathbf{4 j}$ & 2-furyl & $\mathrm{Et}$ & 40 & 80 \\
$\mathbf{4 k}$ & 2-thienyl & $\mathrm{Et}$ & 50 & 73 \\
$\mathbf{4 l}$ & 2-thienyl & $\mathrm{Me}$ & 45 & 70 \\
$\mathbf{4 m}$ & 1-naphthyl & $\mathrm{Et}$ & 60 & 85 \\
\hline
\end{tabular}

${ }^{a}$ All reactions were performed with $\mathbf{1}, \mathbf{2}$, and $\mathbf{3}$ (1 mmol of each); ${ }^{\mathrm{b}}$ Isolated pure yields.

The structures of all the synthesized thiazolopyridone derivatives 4 were deduced by their satisfactory spectral $\left({ }^{1} \mathrm{H},{ }^{13} \mathrm{C}\right.$ NMR and mass) studies and explicitly established by the single 
crystal X-ray diffraction analysis (see the ESI) of one representative compound, 4a (CCDC947810) (Figure 1).
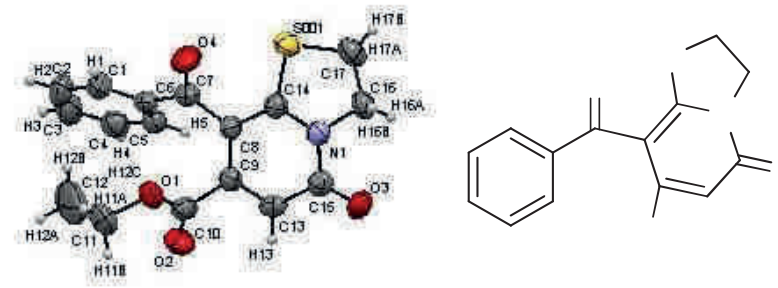

Figure1. ORTEP diagram of $\mathbf{4 a}$.

By taking our entire experimental outcomes into consideration, a plausible reaction mechanism accounting for the formation of compound $\mathbf{4}$ is outlined in Scheme 2. The first step is believed to be the formation of cyclic $N, S$-acetal $\mathbf{A}$ by the reaction of $\alpha$-enolic dithioester 1 with cysteamine 2. The cyclic $N, S$-acetal $\mathbf{A}$ has been isolated and fully characterized. ${ }^{39}$ Next, conjugate addition of $N, S$-acetal $\mathbf{A}$ to dialkyl acetylenedicarboxylate $\mathbf{3}$ gave an intermediate $\mathbf{B}$, which rapidly undergoes intramolecular regioselective $\mathrm{N}$-cyclization to furnish the desired bicyclic thiazolopyridone 4. During the course of our investigation, we could not observe the formation of pyrrolothiazolone 5 even in a trace.<smiles>C=C(C)/C=C(/C)C(=C)C</smiles>

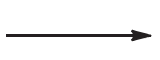

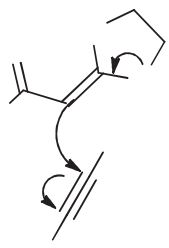
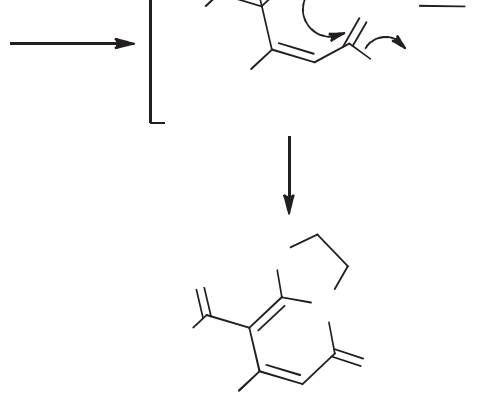

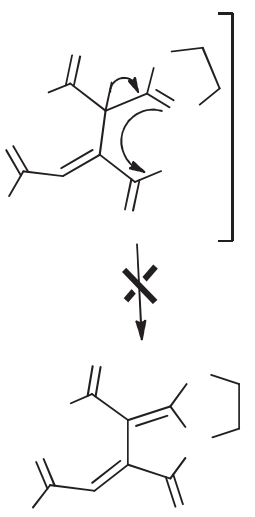

Scheme 2. Plausible mechanism for the formation of thiazolopyridones 4.

\section{Conclusions}

In summary, we have developed an operationally simple and straightforward one-pot multicomponent reaction involving dithioesters, cysteamine and dialkyl acetylenedicarboxylate under metal-free and solvent-free conditions for the first time. The reaction resulted in a convenient synthesis of diverse highly functionalized thiazolopyridones in high yields from 
acyclic precursors. This convergent and highly regioselective approach exhibits an unusually high multiple $\mathrm{C}-\mathrm{C}, \mathrm{C}-\mathrm{N}$ and $\mathrm{C}-\mathrm{S}$ bond forming efficiency utilizing all the reactants efficiently in structure, cost, and step economies. It is noteworthy that the reaction tolerates a broad range of functional groups. Significantly, the presence of keto-group and ester groups makes these compounds excellent entrants as precursors for further synthetic renovations. We hope this clean and green MCC protocol may be of immense value for both synthetic and medicinal chemists.

\section{Experimental Section}

The commercially available starting materials were used as received without further purification. $\alpha$-Enolic dithioesters 1 were prepared by the reported procedure. ${ }^{21}{ }^{1} \mathrm{H}$ and ${ }^{13} \mathrm{C}$ NMR spectra were recorded on NMR spectrometers operating at 300 and $75 \mathrm{MHz}$, respectively. Chemical shifts $(\delta)$ are given in parts per million ( $\mathrm{ppm}$ ) using the residual solvent peaks as reference relative to TMS. $J$ values are given in Hz.

General procedure for the synthesis of thiazolopyridones (4). A round bottom flask was charged with the appropriate $\alpha$-enolic dithioesters $1(1 \mathrm{mmol})$, cysteamine $2(1 \mathrm{mmol})$ and placed in a pre-heated oil bath at $110{ }^{\circ} \mathrm{C}$. The reaction mixture was heated for $15 \mathrm{~min}$ until formation of the cyclic N,S-acetal (monitored by TLC) was complete, then the temperature was reduced to 90 ${ }^{\circ} \mathrm{C}$ and dialkyl acetylenedicarboxylate $3(1 \mathrm{mmol})$ was added. The reaction mixture was stirred for the stipulated time (see Table 2). After completion of the reaction (TLC), the reaction was quenched with water and the solution was extracted with DCM followed by washing with brine. The organic layer was dried $\left(\mathrm{Na}_{2} \mathrm{SO}_{4}\right)$ and concentrated in vacuo. The resultant reaction mixture was purified by column chromatography using silica gel as stationary phase and ethyl acetatehexane (3:7) as eluent to afford the analytically pure product 4.

Ethyl 8-benzoyl-5-oxo-2,3-dihydro-5H-thiazolo[3,2-a]pyridine-7-carboxylate (4a). White solid (0.223 g, $87 \%)$; mp 160-162 ${ }^{\circ} \mathrm{C} .{ }^{1} \mathrm{H}$ NMR (300 MHz, $\left.\mathrm{CDCl}_{3}\right): \delta 7.65(\mathrm{~d}, J 6.9 \mathrm{~Hz}, 2 \mathrm{H})$, $7.53(\mathrm{t}, J 7.2 \mathrm{~Hz}, 1 \mathrm{H}), 7.43(\mathrm{t}, J 7.2 \mathrm{~Hz}, 2 \mathrm{H}), 6.63(\mathrm{~s}, 1 \mathrm{H}), 4.56(\mathrm{t}, J 7.8 \mathrm{~Hz}, 2 \mathrm{H}), 3.66$ (q, $J 7.2$ $\mathrm{Hz}, 2 \mathrm{H}), 3.37(\mathrm{t}, J 7.5 \mathrm{~Hz}, 2 \mathrm{H}), 0,98(\mathrm{t}, J 7.2 \mathrm{~Hz}, 3 \mathrm{H}) .{ }^{13} \mathrm{C} \mathrm{NMR}\left(75 \mathrm{MHz}, \mathrm{CDCl}_{3}\right): \delta 191.9$, $165.1,160.4,156.9,143.8,138.2,132.5,128.5,128.3,115.9,110.5,62.0,50.6,28.1,13.3$. IR $\left(\mathrm{KBr}, \mathrm{cm}^{-1}\right): 3048,2983,1720,1673,1634,1485,1270,1063$. HRMS $\left(\mathrm{ESI}^{+}\right)$: calcd for $\mathrm{C}_{17} \mathrm{H}_{15} \mathrm{NO}_{4} \mathrm{~S}\left[\mathrm{M}+\mathrm{H}^{+}\right], 330.0795$; found, 330.0807 .

Methyl 8-benzoyl-5-oxo-2,3-dihydro-5H-thiazolo[3,2-a]pyridine-7-carboxylate (4b). White solid (0.252 g, $80 \%)$; mp 190-192 ${ }^{\circ} \mathrm{C} .{ }^{1} \mathrm{H}$ NMR (300 MHz, $\left.\mathrm{CDCl}_{3}\right): \delta 7.62(\mathrm{~d}, J 7.2 \mathrm{~Hz}, 2 \mathrm{H})$, $7.51(\mathrm{~d}, J 7.2 \mathrm{~Hz}, 1 \mathrm{H}), 7.46-7.41(\mathrm{~m}, 2 \mathrm{H}), 6.61(\mathrm{~s}, 1 \mathrm{H}), 4.56(\mathrm{t}, J 7.8 \mathrm{~Hz}, 2 \mathrm{H}), 3.37$ (t, $J 7.8 \mathrm{~Hz}$, 2H), $3.23(\mathrm{~s}, 3 \mathrm{H}) .{ }^{13} \mathrm{C} \mathrm{NMR}\left(75 \mathrm{MHz}, \mathrm{CDCl}_{3}\right): \delta 192.0,169.1,165.7,160.4,157.2,143.6,138.4$, $132.5,128.6,128.2,116.0,110.6,52.3,50.7,28.2$. HRMS $\left(\mathrm{ESI}^{+}\right)$: calcd for $\mathrm{C}_{16} \mathrm{H}_{13} \mathrm{NO}_{4} \mathrm{~S}$ $\left[\mathrm{M}+\mathrm{H}^{+}\right], 316.0638$; found, 316.0655 . 
Ethyl 8-(4-methylbenzoyl)-5-oxo-2,3-dihydro-5 $H$-thiazolo[3,2-a]pyridine-7-carboxylate (4c). White solid (0.315 g, $92 \%$ ); mp 152-153 ${ }^{\circ} \mathrm{C} .{ }^{1} \mathrm{H}$ NMR $\left(300 \mathrm{MHz}, \mathrm{CDCl}_{3}\right): \delta 7.56(\mathrm{~d}, J 7.8 \mathrm{~Hz}$, 2H), $7.22(\mathrm{~d}, J 7.5 \mathrm{~Hz}, 2 \mathrm{H}), 6.62(\mathrm{~s}, 1 \mathrm{H}), 4.55(\mathrm{t}, J 7.5 \mathrm{~Hz}, 2 \mathrm{H}), 3.70(\mathrm{q}, J 6.9 \mathrm{~Hz}, 2 \mathrm{H}), 3.36(\mathrm{t}, J$ $7.5 \mathrm{~Hz}, 2 \mathrm{H}), 2.39$ (s, 3H), 0.99 (t, $J 6.9 \mathrm{~Hz}, 3 \mathrm{H}) .{ }^{13} \mathrm{C} \mathrm{NMR}\left(75 \mathrm{MHz}, \mathrm{CDCl}_{3}\right): \delta 191.7,165.2$, $160.5,156.2$, 143.8, 143.5, 135.5, 129.2, 128.5, 116.0, 115.9, 110.9, 62.0, 50.6, 28.2, 21.5, 13.3. HRMS (ESI ${ }^{+}$): calcd for $\mathrm{C}_{18} \mathrm{H}_{17} \mathrm{NO}_{4} \mathrm{~S}\left[\mathrm{M}+\mathrm{H}^{+}\right], 344.0951$; found, 344.0973.

Ethyl 8-(4-methoxybenzoyl)-5-oxo-2,3-dihydro-5H-thiazolo[3,2-a]pyridine-7-carboxylate (4d). Brown solid (0.294 g, 82 \%); mp 108-110 ${ }^{\circ} \mathrm{C} .{ }^{1} \mathrm{H}$ NMR (300 MHz, $\mathrm{CDCl}_{3}$ ): $\delta 7.65$ (d, $J 8.1$ $\mathrm{Hz}, 2 \mathrm{H}), 6.91(\mathrm{~d}, J 8.1 \mathrm{~Hz}, 2 \mathrm{H}), 6.64(\mathrm{~s}, 1 \mathrm{H}), 4.54(\mathrm{t}, J 7.2 \mathrm{~Hz}, 2 \mathrm{H}), 3.85-3.77(\mathrm{~m}, 5 \mathrm{H}), 3.37(\mathrm{t}, J$ $7.2 \mathrm{~Hz}, 2 \mathrm{H}), 1.01(\mathrm{t}, J 6.9 \mathrm{~Hz}, 3 \mathrm{H}) .{ }^{13} \mathrm{C}$ NMR $\left(75 \mathrm{MHz}, \mathrm{CDCl}_{3}\right): \delta 190.7,165.1,163.2,160.4$, 155.5, 143.6, 130.6, 115.8, 115.7, 113.6, 111.6, 61.9, 55.3, 50.6, 28.2, 13.2. HRMS (ESI $\left.{ }^{+}\right)$: calcd for $\mathrm{C}_{18} \mathrm{H}_{17} \mathrm{NO}_{5} \mathrm{~S}\left[\mathrm{M}+\mathrm{H}^{+}\right], 360.0900$; found, 360.0915 .

Methyl 8-(3-methoxybenzoyl)-5-oxo-2,3-dihydro-5H-thiazolo[3,2-a]pyridine-7-carboxylate (4e). Brown solid (0.293 g, $85 \%$ ); mp 110-112 ${ }^{\circ} \mathrm{C} .{ }^{1} \mathrm{H}$ NMR (300 MHz, $\left.\mathrm{CDCl}_{3}\right): \delta 7.35-7.26(\mathrm{~m}$, 1H), 7.19-7.05 (m, 3H), $6.61(\mathrm{~s}, 1 \mathrm{H}), 4.57(\mathrm{t}, J 7.5 \mathrm{~Hz}, 2 \mathrm{H}), 3.84(\mathrm{~s}, 3 \mathrm{H}) 3.38(\mathrm{t}, J 7.5 \mathrm{~Hz}, 2 \mathrm{H})$, $3.28(\mathrm{~s}, 3 \mathrm{H}) .{ }^{13} \mathrm{C}$ NMR $\left(75 \mathrm{MHz}, \mathrm{CDCl}_{3}\right): \delta 191.5,165.5,160.3,159.6,157.3,143.5,139.4$, 129.5, 120.6, 119.2, 115.8, 115.7, 112.2, 110.5, 55.2, 52.2, 50.6, 28.1. HRMS (ESI ${ }^{+}$): calcd for $\mathrm{C}_{17} \mathrm{H}_{15} \mathrm{NO}_{5} \mathrm{~S}\left[\mathrm{M}+\mathrm{H}^{+}\right], 346.0744$; found, 346.0760.

Methyl 8-(1,3-benzodioxol-5-ylcarbonyl)-5-oxo-2,3-dihydro-5 $\boldsymbol{H}$-thiazolo[3,2-a]pyridine-7carboxylate (4f). Yellow solid (0.323 g, $90 \%)$; mp 145-147 ${ }^{\circ} \mathrm{C} .{ }^{1} \mathrm{H}$ NMR $\left(300 \mathrm{MHz}, \mathrm{CDCl}_{3}\right)$ : $\delta 7.22(\mathrm{~s}, 1 \mathrm{H}), 7.12(\mathrm{~d}, J 7.8 \mathrm{~Hz}, 1 \mathrm{H}), 6.79(\mathrm{~d}, J 8.1 \mathrm{~Hz}, 1 \mathrm{H}), 6.61(\mathrm{~s}, 1 \mathrm{H}), 6.04(\mathrm{~s}, 2 \mathrm{H}), 4.54(\mathrm{t}, J$ $7.8 \mathrm{~Hz}, 2 \mathrm{H}), 3.44(\mathrm{~s}, 3 \mathrm{H}), 3.37$ (t, $J 7.5 \mathrm{~Hz}, 2 \mathrm{H}) .{ }^{13} \mathrm{C} \mathrm{NMR}\left(75 \mathrm{MHz}, \mathrm{CDCl}_{3}\right): \delta 190.3,165.4$, $160.3,155.6,151.4,148.1,143.2,132.5,124.6,115.87,115.81,110.8,107.9,107.6,101.8,52.4$, 50.6, 28.2. HRMS (ESI ${ }^{+}$): calcd for $\mathrm{C}_{17} \mathrm{H}_{13} \mathrm{NO}_{6} \mathrm{~S}\left[\mathrm{M}+\mathrm{H}^{+}\right], 360.0536$; found, 360.0551 .

Methyl 5-oxo-8-[4-(trifluoromethyl)benzoyl]-2,3-dihydro-5H-thiazolo[3,2-a]pyridine-7carboxylate (4g). Light yellow solid (0.287 g, $75 \%$ ); mp 196-197 ${ }^{\circ} \mathrm{C} .{ }^{1} \mathrm{H}$ NMR $(300 \mathrm{MHz}$, $\left.\mathrm{CDCl}_{3}\right): \delta 7.74-7.68(\mathrm{~m}, 4 \mathrm{H}), 6.62(\mathrm{~s}, 1 \mathrm{H}), 4.58(\mathrm{t}, J 7.8 \mathrm{~Hz}, 2 \mathrm{H}), 3.40(\mathrm{t}, J 7.8 \mathrm{~Hz}, 2 \mathrm{H}), 3.26(\mathrm{~s}$, $3 \mathrm{H}) .{ }^{13} \mathrm{C}$ NMR $\left(75 \mathrm{MHz}, \mathrm{CDCl}_{3}\right): \delta 190.6,165.3,160.1,158.1,143.1,141.3,133.5$ (q, J $32.7 \mathrm{~Hz}$ 1C), $128.3,125.4$ (q, $J 3.75 \mathrm{~Hz}, 1 \mathrm{C}), 121.5,116.1,109.7,52.3,50.6,28.1 . \mathrm{IR}\left(\mathrm{KBr}, \mathrm{cm}^{-1}\right): 2952$, $1715,1670,1640,1445,1337,1274,1123,1107,1072$. HRMS $\left(E S I^{+}\right)$: calcd for $\mathrm{C}_{17} \mathrm{H}_{12} \mathrm{~F}_{3} \mathrm{NO}_{4} \mathrm{~S}$ $\left[\mathrm{M}+\mathrm{H}^{+}\right], 384.0512$; found, 384.0527 .

Ethyl 8-(2-chlorobenzoyl)-5-oxo-2,3-dihydro-5 $H$-thiazolo[3,2-a]pyridine-7-carboxylate (4h). Brown solid (0.272 g, $75 \%$ ); mp 183-185 ${ }^{\circ} \mathrm{C} .{ }^{1} \mathrm{H}$ NMR (300 MHz, $\mathrm{CDCl}_{3}$ ): $\delta 7.44-7.28(\mathrm{~m}, 4 \mathrm{H})$, $6.43(\mathrm{~s}, 1 \mathrm{H}), 4.55(\mathrm{t}, J 7.8 \mathrm{~Hz}, 2 \mathrm{H}), 3.71(\mathrm{q}, 7.2 \mathrm{~Hz}, 2 \mathrm{H}), 3.37$ (t, $J 7.8 \mathrm{~Hz}, 2 \mathrm{H}), 1.11(\mathrm{t}, J 7.2 \mathrm{~Hz}$, $3 \mathrm{H}) .{ }^{13} \mathrm{C}$ NMR $\left(75 \mathrm{MHz} \mathrm{CDCl}_{3}\right): \delta 189.3,165.5,160.3,159.6,144.7,137.6,132.5,131.9$, $130.5,130.0,126.5,115.5,115.4,62.1,50.5,28.1,13.6$. IR $\left(\mathrm{KBr}, \mathrm{cm}^{-1}\right): 2978,1730,1669,1620$, 1479, 1262. HRMS (ESI ${ }^{+}$): calcd for $\mathrm{C}_{17} \mathrm{H}_{14} \mathrm{ClNO}_{4} \mathrm{~S}\left[\mathrm{M}+\mathrm{H}^{+}\right], 364.0405$; found, 364.0418.

Methyl 8-(2-chlorobenzoyl)-5-oxo-2,3-dihydro-5H-thiazolo [3,2-a]pyridine-7-carboxylate (4i). Brown solid (0.244 g, $70 \%$ ); mp 181-183 ${ }^{\circ} \mathrm{C} .{ }^{1} \mathrm{H}$ NMR (300 MHz, $\left.\mathrm{CDCl}_{3}\right): \delta 7.43-7.30(\mathrm{~m}$, $4 \mathrm{H}), 6.43(\mathrm{~s}, 1 \mathrm{H}), 4.55(\mathrm{t}, J 7.5 \mathrm{~Hz}, 2 \mathrm{H}), 3.36-3.28(\mathrm{~m}, 5 \mathrm{H}) .{ }^{13} \mathrm{C}$ NMR $\left(75 \mathrm{MHz}, \mathrm{CDCl}_{3}\right)$ : 
$\delta 189.2,165.9,160.1,159.8,144.1,137.6,132.3,131.8,130.5,129.7,126.5,115.5,115.3,110.6$, 52.4, 50.4, 28.0. HRMS (ESI ${ }^{+}$): calcd for $\mathrm{C}_{16} \mathrm{H}_{12} \mathrm{ClNO}_{4} \mathrm{~S}\left[\mathrm{M}+\mathrm{H}^{+}\right]$, 350.0248; found, 350.0262.

Ethyl 8-(furan-2-ylcarbonyl)-5-oxo-2,3-dihydro-5H-thiazolo[3,2-a]pyridine-7-carboxylate (4j). Brown solid (0.255 g, $80 \%$ ); mp 120-122 ${ }^{\circ} \mathrm{C} .{ }^{1} \mathrm{H}$ NMR (300 MHz, $\left.\mathrm{CDCl}_{3}\right): \delta 7.52(\mathrm{~s}, 1 \mathrm{H})$, $7.16(\mathrm{~d}, J 3.6 \mathrm{~Hz}, 1 \mathrm{H}), 6.74(\mathrm{~s}, 1 \mathrm{H}), 6.54(\mathrm{~s}, 1 \mathrm{H}), 4.55(\mathrm{t}, J 7.8 \mathrm{~Hz}, 2 \mathrm{H}), 3.98(\mathrm{q}, J 7.2 \mathrm{~Hz}, 2 \mathrm{H})$, $3.35(\mathrm{t}, J 7.8 \mathrm{~Hz}, 2 \mathrm{H}), 1.08(\mathrm{t}, J 7.2 \mathrm{~Hz}, 3 \mathrm{H}) .{ }^{13} \mathrm{C} \mathrm{NMR}\left(75 \mathrm{MHz}, \mathrm{CDCl}_{3}\right): \delta 178.7,165.1,160.4$, 156.7, 152.6, 145.9, 145.8, 143.0, 117.2, 116.6, 116.5, 112.6, 112.5, 110.0, 62.1, 50.6, 28.1, 13.4 . IR $\left(\mathrm{KBr}, \mathrm{cm}^{-1}\right): 2984,1723,1665,1631,1458,1425,1257$. HRMS $\left(\mathrm{ESI}^{+}\right)$: calcd for $\mathrm{C}_{15} \mathrm{H}_{13} \mathrm{NO}_{5} \mathrm{~S}$ $\left[\mathrm{M}+\mathrm{H}^{+}\right], 320.0587$; found, 320.0600 .

Ethyl 5-oxo-8-(thiophen-2-ylcarbonyl)-2,3-dihydro-5 $\boldsymbol{H}$-thiazolo[3,2-a]pyridine-7-carboxylate (4k). Brown solid (0.259 g, $73 \%$ ); mp 130-132 ${ }^{\circ} \mathrm{C} .{ }^{1} \mathrm{H}$ NMR $\left(300 \mathrm{MHz}, \mathrm{CDCl}_{3}\right): \delta 7.66(\mathrm{~d}$, $5.1 \mathrm{~Hz}, 1 \mathrm{H}), 7.35(\mathrm{~d}, J 3 \mathrm{~Hz}, 1 \mathrm{H}), 7.08-7.05(\mathrm{~m}, 1 \mathrm{H}), 6.69(\mathrm{~s}, 1 \mathrm{H}), 4.56(\mathrm{t}, J 7.5 \mathrm{~Hz}, 2 \mathrm{H}), 3.88$ (q, $J 6.9 \mathrm{~Hz}, 2 \mathrm{H}), 3.39(\mathrm{t}, J 7.2 \mathrm{~Hz}, 2 \mathrm{H}), 1.04(\mathrm{t}, J 6.9 \mathrm{~Hz}, 3 \mathrm{H}) .{ }^{13} \mathrm{C} \mathrm{NMR}\left(75 \mathrm{MHz}, \mathrm{CDCl}_{3}\right): \delta 183.8$, 165.0, 160.3, 155.4, 144.1, 143.0, 133.9, 132.4, 127.7, 116.4, 116.3, 110.8, 62.1, 50.7, 28.3, 13.3. HRMS (ESI ${ }^{+}$): calcd for $\mathrm{C}_{15} \mathrm{H}_{13} \mathrm{NO}_{4} \mathrm{~S}_{2}\left[\mathrm{M}+\mathrm{H}^{+}\right]$, 336.0359; found, 336.0374.

Methyl 5-oxo-8-(thiophene-2-carbonyl)-2,3-dihydro-5 $\boldsymbol{H}$-thiazolo[3,2-a]pyridine-7-carboxylate (4I). White solid $(0.224 \mathrm{~g}, 70 \%)$; mp $129-131^{\circ} \mathrm{C} .{ }^{1} \mathrm{H} \mathrm{NMR}\left(300 \mathrm{MHz}, \mathrm{CDCl}_{3}\right): \delta 7.66(\mathrm{~d}, J$ $4.8 \mathrm{~Hz}, 1 \mathrm{H}), 7.32(\mathrm{~d}, J 3.3 \mathrm{~Hz}, 1 \mathrm{H}), 7.07(\mathrm{t}, J 4.5 \mathrm{~Hz}, 1 \mathrm{H}), 6.67(\mathrm{~s}, 1 \mathrm{H}), 4.56(\mathrm{t}, J 7.5 \mathrm{~Hz}, 2 \mathrm{H})$, 3.44-3.37 (m, 7H). ${ }^{13} \mathrm{C}$ NMR (75 MHz, $\left.\mathrm{CDCl}_{3}\right): \delta 183.9,165.6,160.4,155.7,144.0,142.8$, $133.8,132.2,127.7,116.5,116.4,110.9,52.5,50.8,28.3$. IR $\left(\mathrm{KBr}, \mathrm{cm}^{-1}\right): 2925,1715,1658$, 1630, 1497, 1441, 1415, 1270. HRMS $\left(\mathrm{ESI}^{+}\right)$: calcd for $\mathrm{C}_{14} \mathrm{H}_{11} \mathrm{NO}_{4} \mathrm{~S}_{2}\left[\mathrm{M}+\mathrm{H}^{+}\right], 322.0202$; found, 322.0215 .

Ethyl 8-(1-naphthoyl)-5-oxo-2,3-dihydro-5H-thiazolo[3,2-a]pyridine-7-carboxylate (4m). White solid (0.322 g, $85 \%$ ); mp 218-220 ${ }^{\circ} \mathrm{C} .{ }^{1} \mathrm{H}$ NMR $\left(300 \mathrm{MHz}, \mathrm{CDCl}_{3}\right): \delta 8.45(\mathrm{~d}, J 8.1 \mathrm{~Hz}$, 1H), 7.95-7.85 (m, 2H), 7.63-7.54 (m, 2H), $7.42(\mathrm{~s}, 2 \mathrm{H}), 6.49(\mathrm{~s}, 1 \mathrm{H}), 4.56(\mathrm{t}, J 7.2 \mathrm{~Hz}, 2 \mathrm{H}), 3.36$ $(\mathrm{t}, J 8.1 \mathrm{~Hz}, 2 \mathrm{H}), 3.22(\mathrm{~s}, 2 \mathrm{H}), 0.68(\mathrm{t}, J 7.2 \mathrm{~Hz}, 3 \mathrm{H}) .{ }^{13} \mathrm{C} \mathrm{NMR}\left(75 \mathrm{MHz}, \mathrm{CDCl}_{3}\right): \delta 192.3$, $165.6,160.4,158.8,145.0,135.7,133.8,132.3,130.5,128.2,127.8,126.7,125.8,124.0,115.5$, 115.4, 61.8, 50.5, 28.1, 13.0. HRMS $\left(\mathrm{ESI}^{+}\right)$: calcd for $\mathrm{C}_{21} \mathrm{H}_{17} \mathrm{NO}_{4} \mathrm{~S}\left[\mathrm{M}+\mathrm{Na}^{+}\right]$, 402.0770; found, 402.0778 .

\section{Acknowledgements}

We gratefully acknowledge the generous financial support of the Science and Engineering Research Board (Grant No. SB/S1/OC-30/2013) and the Council of Scientific and Industrial Research (Grant No. 02(0072)/12/EMR-II), New Delhi, India. A.N. and G.K.V. are thankful to the UGC, and B.J.R. G.S. and A.S. are thankful to the CSIR, New Delhi, for research fellowships. 


\section{References}

1. Tietze, L. F. Chem. Rev. 1996, 96, 115. http://dx.doi.org/10.1021/cr950027e

2. Tietze, L. F.; Brasche, G.; Gericke, K. M. Domino Reactions in Organic Synthesis, WileyVCH, Weinheim, Germany, 2006. http://dx.doi.org/10.1002/9783527609925

3. Coquerel, Y.; Boddaert, T.; Presset, M.; Mailhol D.; Rodriguez, J. In Ideas in Chemistry and Molecular Sciences: Advances in Synthetic Chemistry, Pignataro, B. ed.; Wiley-VCH: Weinheim, Germany, 2010, Vol. I, p 187. http://dx.doi.org/10.1002/9783527630554.ch9

4. Pellissier, H. Chem. Rev. 2013, 113, 442. http://dx.doi.org/10.1021/cr300271k

5. Pozharskii, A. F.; Soldatenkov, A.; Katritzky, A. R. Heterocycles in life and society: An introduction to heterocyclic chemistry, biochemistry and applications, John Wiley \& Sons, Edn., 2011. http://dx.doi.org/10.1002/9781119998372

6. Li, J. J. Heterocyclic Chemistry in Drug Discovery, John Wiley \& Sons, 2013.

7. For a special issue, see: Chem. Rev. 2004, 104, Issue 5.

8. Kale, R.R.; Kale, M.G.; Waterson, D.; Raichurkar, A.; Hameed, S.P.; Manjunatha, M.R.; Reddy, B.K.K.; Malolanarasimhan, K.; Shinde, V.; Koushik, K.; Jena, L.K.; Menasinakai, S.; Humnabadkar, V.; Madhavapeddi, P.; Basavarajappa, H.; Sharma, S.; Nandishaiah, R.; Kumar, K.N.M.; Ganguly, S.; Ahuja, V.; Gaonkar, S.; Kumar, C.N.N.; Ogg, D.; BoriackSjodin, P.A.; Sambandamurthy, V.K.; de Sousa, S.M.; Ghorpade, S.R. Bioorg. Med. Chem. Lett. 2014, 24, 870. http://dx.doi.org/10.1016/j.bmcl.2013.12.080

9. Kale, M.G.; Raichurkar, A.; Hameed, S.P.; Waterson, D.; McKinney, D.; Manjunatha, M.R.; Kranthi, U.; Koushik, K.; Jena, L.K.; Shinde, V.; Rudrapatna, S.; Barde, S.; Humnabadkar, V.; Madhavapeddi, P.; Basavarajappa, H.; Ghosh, A.; Ramya, V.K.; Guptha, S, Sharma S, Vachaspati P, Kumar KN, Giridhar J, Reddy J, Panduga V, Ganguly S, Ahuja V.; Gaonkar, S.; Kumar, C.N.N.; Ogg, D.; Tucker, J.A.; Boriack-Sjodin, P.A.; de Sousa, S.M.;

Sambandamurthy, V.K.; Ghorpade, S.R. J. Med. Chem. 2013, 56, 8834. http://dx.doi.org/10.1021/jm401268f

10. Emtenas, H.; Ahlin, K.; Pinkner, J. S.; Hultgren, S. J.; Almqvist, F. J. Comb. Chem. 2002, 4, 630. http://dx.doi.org/10.1021/cc020032d

11. Chorell, E.; Pinkner, J. S.; Phan, G.; Edvinsson, S.; Buelens, F.; Remaut, H.; Waksman, G.; Hultgren, S. J.; Almqvist, F. J. Med. Chem. 2010, 53, 5690.

http://dx.doi.org/10.1021/jm100470t 
12. Sellstedt, M.; Dang, H. T.; Prasad, G. K.; Sauer, U.; Almqvist, F. Eur. J. Org. Chem. 2013, 7476. http://dx.doi.org/10.1002/ejoc.201301371

13. Chorell, E.; Das, P.; Almqvist, F. J. Org. Chem. 2007, 72, 4917. http://dx.doi.org/10.1021/jo0704053

14. Bengtsson, C.; Almqvist, F. J. Org. Chem. 2010, 75, 972. http://dx.doi.org/10.1021/jo902458g

15. Bengtsson, C.; Almqvist, F. J. Org. Chem. 2011, 76, 9817. http://dx.doi.org/10.1021/jo201952p

16. Sellstedt, M.; Almqvist, F. Org. Lett., 2008, 10, 4005. http://dx.doi.org/10.1021/o1801506y

17. Chiba, T.; Takahashi, T.; Kaneko, C. Chem. Pharm. Bull. 1985, 33, 4002. http://dx.doi.org/10.1248/cpb.33.4002

18. Britsun, V. N.; Esipenko, A. N.; Gootov, A. V.; Chernega, A. N.; Lozinskii, M. O. Chem. Het. Comp., 2009, 45, 445. http://dx.doi.org/10.1007/s10593-009-0284-9

19. Pemberton, N.; Aberg, V.; Almstedt, H.; Westermark, A.; Almqvist, F. J. Org. Chem. 2004, 69, 7830. http://dx.doi.org/10.1021/jo048554y

20. Silva, H. I. D.; Henry, W. P.; Pittman Jr, C. U. Synthesis 2012, 44, 3337. http://dx.doi.org/10.1055/s-0032-1316790

21. Samuel, R.; Asokan, C. V.; Suma, S.; Chandran, P.; Retnamma, S.; Anabha, E. R. Tetrahedron Lett. 2007, 48, 8376. http://dx.doi.org/10.1016/j.tetlet.2007.09.076

22. Singh, O. M.; Devi, N. S.; Thokchom, D. S.; Sharma, G. J. Eur. J. Med. Chem. 2010, 45, 2250 . http://dx.doi.org/10.1016/j.ejmech.2010.01.070

23. Peruncheralathan, S.; Khan, T. A.; Ila, H.; Junjappa, H. J. Org. Chem. 2005, 70, 10030. http://dx.doi.org/10.1021/jo051771u

24. Li, M.; Cao, H.; Wang, Y.; Lv, X.-L.; Wen, L.-R. Org. Lett. 2012, 14, 3470. http://dx.doi.org/10.1021/o1301441v

25. Wen, L.-R.; Li, Z.-R.; Li, M.; Cao, H. Green Chem. 2012, 14, 707. http://dx.doi.org/10.1039/c2gc16388h

26. Nagaraju, A.; Ramulu, B. J.; Shukla, G.; Srivastava, A.; Verma, G. K.; Raghuvanshi, K.; Singh, M. S. Green Chem. 2015, 17, 950. http://dx.doi.org/10.1039/C4GC01431F

27. Singh, M. S., Nagaraju, A.; Verma, G. K.; Shukla, G.; Verma, R. K.; Srivastava, A.; Raghuvanshi, K. Green Chem. 2013, 15, 954. http://dx.doi.org/10.1039/c3gc37047j 
28. Nagaraju, A.; Shukla, G.; Srivastava, A.; Ramulu, B. J.; Verma, G. K.; Raghuvanshi, K.; Singh, M. S. Tetrahedron 2014, 70, 3740. http://dx.doi.org/10.1016/j.tet.2014.03.097

29. Shukla, G.; Nagaraju, A.; Srivastava, A.; Verma, G. K.; Raghuvanshi, K.; Singh, M. S. Synthesis 2014, 46, 1815. http://dx.doi.org/10.1055/s-0033-1341086

30. Nandi, G. C.; Samai, S.; Singh, M. S. J. Org. Chem. 2011, 76, 8009. http://dx.doi.org/10.1021/jo200685e

31. Nandi, G. C.; Singh, M. S.; Ila, H.; Junjappa, H. Eur. J. Org. Chem. 2012, 967. http://dx.doi.org/10.1002/ejoc.201101397

32. Verma, R. K.; Verma, G. K.; Raghuvanshi, K.; Singh, M. S. Tetrahedron 2011, 67, 584. http://dx.doi.org/10.1016/j.tet.2010.11.055

33. Kumar, R.; Raghuvanshi, K.; Verma, R. K.; Singh, M. S. Tetrahedron Lett. 2010, 51, 5933. http://dx.doi.org/10.1016/j.tetlet.2010.09.017

34. Verma, G. K.; Raghuvanshi, K.; Verma, R. K.; Dwivedi, P.; Singh, M. S. Tetrahedron 2011, 67, 3698. http://dx.doi.org/10.1016/j.tet.2011.03.078

35. Shukla, G.; Verma, R. K.; Verma, G. K.; Singh, M. S. Tetrahedron Lett. 2011, 52, 7195. http://dx.doi.org/10.1016/j.tetlet.2011.10.136

36. Singh, M. S.; Nandi, G. C.; Samai, S. Green Chem. 2012, 14, 447. http://dx.doi.org/10.1039/c1gc16129f

37. Verma, R. K.; Verma, G. K.; Shukla, G.; Singh, M. S. RSC Adv. 2012, 2, 2413. http://dx.doi.org/10.1039/c2ra00987k

38. Chanda, T.; Verma, R. K.; Singh, M. S. Chem. - Asian J. 2012, 7, 778. http://dx.doi.org/10.1002/asia.201100872

39. Huang, Z.-T.; Shi, X. Synthesis 1990, 162. http://dx.doi.org/10.1055/s-1990-26822 